\title{
Aislamiento territorial y expectativas sociales sobre los hijos: el caso de la caleta Paposo
}

\author{
Luis Miguel Rodrigo \\ Universidad Católica del Norte, Antofagasta, Chile \\ Email: lrodrigo@ucn.cl \\ Emilio Ricci \\ Universidad Católica del Norte, Antofagasta, Chile \\ Email: ericci@ucn.cl
}

\begin{abstract}
Resumen: El aislamiento territorial afecta a distintas dimensiones del desarrollo de las poblaciones que lo padecen. Sus efectos en la materialidad son los más visibles, el déficit de infraestructuras, de servicios públicos y de conectividad reducen claramente el bienestar social de las poblaciones aisladas. Sin embargo, las consecuencias en la subjetividad de estas poblaciones, aunque menos visibles que lasprimeras, pueden ser más importantes. Este trabajo analiza cómo las expectativas sociales que tienen las familias sobre sus hijos, se ven afectadas por una situación de aislamiento territorial. Para ello, se realizó una encuesta a dos grupos de madres de alumnos de la Región de Antofagasta. El primer grupo reside en un territorio aislado de la región,la caleta Paposo, mientras que el segundo grupo reside en la capital regional, Antofagasta. Los resultados muestran que las madres del territorio aislado tienen menores expectativas económicas y mayores expectativas de migración sobre sus hijos.

Palabras clave: Aislamiento territorial, desigualdad espacial, expectativas sociales, efectos subjetivos
\end{abstract}

\section{Territorial isolation and social expectations about the children: the case of the cove Paposo}

\begin{abstract}
Territorial isolation affects different dimensions of the development of the populations that suffer from it. Its effects on materiality are the most visible; the lack of infrastructure, public services and connectivity clearly reduces the social welfare of isolated populations. However, the consequences on the subjectivity of these populations, although less visible than the former, may be more significant. This paper analyses how the social expectations that families have of their children are affected by a situation of territorial isolation. To this end, a survey was conducted among two groups of mothers of students in the Antofagasta Region. The first group resides in an isolated territory of the region, Cove Paposo, while the second group resides in the regional capital, Antofagasta. The results show that mothers in the isolated territory have lower economic expectations and higher expectations of migration for their children.
\end{abstract}

Keywords: territorial isolation, spatial inequality, social expectations 


\section{Isolamento territorial e expectativas sociais sobre as crianças: o caso da colônia Paposo}

Resumo: O isolamento territorial afeta diferentes dimensões do desenvolvimento das populações que sofrem com isso. Seus efeitos sobre a materialidade são os mais visíveis, o déficit de infraestruturas, serviços públicos e conectividade reduzem claramente o bem-estar social de populações isoladas. Entretanto, as consequências sobre a subjetividade dessas populações, embora menos visíveis que as primeiras, podem ser mais importantes. Este artigo analisa como as expectativas sociais que as famílias têm sobre seus filhos são afetadas por uma situação de isolamento territorial. Para isso, foi realizada uma pesquisa para dois grupos de mães de estudantes da Região de Antofagasta. O primeiro grupo reside em um território isolado da região, a enseada de Paposo, enquanto que o segundo grupo reside na capital regional, Antofagasta. Os resultados mostram que as mães do território isolado têm expectativas econômicas mais baixas e maiores expectativas de migração para seus filhos. sociais

Palavras-chave: Isolamento territorial, desigualdade espacial, expectativas

\section{Introducción}

En la última década, el debate político en Chile se ha desplazado desde el crecimiento económico hacia el problema de la desigualdad social, aunque no todas las dimensiones de la desigualdad han recibido la misma atención. Hasta ahora, la mayor parte del debate político y social se ha centradoen la desigualdad económica, en la desigualdad en la educación y, en menor medida, en la desigualdad en la salud.Frente a éstas, la desigualdad espacial, esto es, aquella generada por la distribución heterogénea de recursos sociales en el territorio nacional, ocupa un lugar secundario o exiguo. Sin embargo, hay que recordar que uno de los grandes problemas de Chile y de Latinoamérica son las dificultades de acceso de una parte de su población a los recursos sociales (servicios públicos y privados, empleos, infraestructuras, etc.) que han tendido a concentrarse históricamente en las grandes ciudades. La concentración espacial de recursos condena a la población más alejada de los grandes centros urbanos a sufrir peores condiciones de vida y menores oportunidades de desarrollo. Que el acceso a los recursos que posibilitan el bienestar social y el desarrollo personal dependa del lugar en el que se nace, es tan injusto,comoel que dependa de la clase social de nuestros padres. Por ello, el Estado chileno tiene la obligación y el desafío de implementar políticas públicas que logren una reducción efectiva de la brecha territorial que sufre el país.

La problemática de la desigualdad espacial tiene múltiples dimensiones. Este artículo tiene como objetivoidentificar las consecuencias del aislamiento territorial en las expectativas sociales que generan las familias sobre sus hijos. En concreto, estudiamos cómo afecta el aislamiento al nivel 
educativo que los padres creen que alcanzarán sus hijos; cómo afecta al nivel ocupacional que los padres creen que alcanzarán sus hijos; y cómo afecta el aislamiento adónde creen los padres que residirán sus hijos, en su misma localidad (no migrarán) o en otra localidad (migrarán)Esperamos, por tanto, y esta es nuestra hipótesis de trabajo, que la situación de aislamiento territorial no sólo afecta al bienestar presente de las familias, sino que reduce además las expectativas del desarrollo futuro de sus miembros más jóvenes, comprometiendo gravemente la sustentabilidad demográfica y socioeconómica de las comunidades aisladas. Junto con Sen $(1985 ; 1995)$ entendemos que la desigualdad no essólo, ni principalmente, un problema ingreso per cápita, sino que pasa fundamentalmente por tener acceso a las distintas formas de vida posibles (libertad positiva). La desigualdad, en este sentido, hay que corregirla en dos niveles: i) en el desarrollo de las capacidades que permiten el acceso de los individuos a las diversas formas de vida (nivel individual); ii) y en las diversas oportunidades objetivas que ofrece el contexto en el que están insertos los individuos (nivel social).

Para comprobar nuestra hipótesis,hemos analizado y comparado las expectativas sociales que tienen sobre sus hijoslas madres de dos colegios de la Región de Antofagasta.El primer colegio se encuentra ubicado en la Caleta Paposo, localidad que fue identificada como aislada por el Gobierno Regional de Antofagasta (GORE). El segundo colegio está ubicado en la ciudad de Antofagasta, considerada por el GORE (2012) como la localidad mejor integrada y conectada de la región. Las madres de ambos colegios fueron encuestadas en el 2013 y los datos producidos por dicha encuesta fueron analizados estadísticamente mediante tablas de contingencia,un modelo lineal general multivariante y un modelo de regresión logística.

Este análisis, novedoso para el caso de Chile, puede contribuir a incorporar una nueva dimensión a la estrategia nacional para el desarrollo de localidades aisladas que se basa fundamentalmente en las condiciones materiales de estas comunidades, sin tener en cuenta de que forma el aislamiento limita las expectativas de desarrollo futuro de las nuevas generaciones.

La organización de este artículo es la siguiente: en el primer apartado realizamos una revisión bibliográfica que da cuenta de la relevancia de las expectativas sociales y de cómo éstas son afectadas por el territorio; posteriormente, explicamos cómo ha sido definido y operacionalizado el concepto de aislamiento territorialen la política pública chilena; después, contextualizamos socio-territorialmente los colegios que serán objeto de estudio; en el apartado metodológico damos cuenta de la población estudio, del instrumento utilizado y de las técnicas estadísticas empleadas; posteriormente, se exponen los resultados estadísticos descriptivos y de dependencia;y, finalmente, se presentan nuestras conclusiones sobre el estudio. 


\section{Marco Teórico}

Es llamativo que, en estos momentos, no exista una literatura específica que aborde los efectos del aislamiento territorial en las expectativas sociales. La línea de trabajo más próxima a este problema estudia las expectativas educativas y laborales de los jóvenes en las zonas rurales, siendo la mayoría de los estudios sobre los casos norteamericano y australiano. Entendemos que ruralidad y aislamiento territorial no son conceptos totalmente equivalentes, aunque la ruralidad suele conllevar un cierto nivel de aislamiento y los territorios aislados se caracterizan por una economía primaria.Pese a las diferencias conceptuales existentes, utilizaremos los estudios rurales para enmarcar teóricamente nuestro trabajo.

Las expectativas sociales de los adolescentes tienen una gran relevancia en el proceso educativo y laboral. Una extensa base empírica nos permite afirmar que son un excelente predictor tanto del logro académico como del profesional, pues son determinantes de las decisiones que realizarán los jóvenes (Beal y Crockett, 2010; Eccles, Vida, y Barber, 2004; Bandura et al., 2001). En la configuración de expectativas intervienen tanto las características propias o internas de los adolescentes cómo su contexto social. Dentro de éste, son especialmente significativas las expectativas que tienen los profesores sobre sus alumnos y, sobre todo, las expectativas que tienen los padres sobre sus hijos (Padilla, Suárez y García, 2007). Pese a que en la adolescencia aparecen otros agentes relevantes (grupo de pares, profesores, medios de comunicación, etc.) la familia sigue siendo considerada el agente de socializaciónprincipal. Por su gran capacidad de influencia, las expectativas familiares son un buen predictor tanto de las aspiraciones de los hijos como de sus trayectorias sociales (Bourdieu y Passeron, 1981; Bourdieu, 1988; Padilla, Suárez y García, 2007).

El principal determinante del nivel educativo y ocupacional que alcanzan los jóvenes es la clase social de sus padres (Bourdieu, 2000; Beck, 1998; Boudon, 1973). Ésta también genera las expectativas sociales de los jóvenes y de sus familias. Los alumnos, mediante las relaciones sociales con sus familiares, profesores y pares (de igual o similar posición social), interiorizan que un determinado nivel educativo es el normal para ellos (el más probable), de esta forma, la probabilidad objetiva se transforma así en expectativas subjetivas, se naturaliza(Criado, 2004; Bourdieu, 1981).

El territorio en el que viven las familias también ha sido identificado como un factor que afecta significativamente la generación de expectativas sociales.Esta línea de estudios se ha centrado fundamentalmente en la comparación entre territoriosurbanos y rurales. Los trabajos realizados en Estados Unidos y Australia, muestran que los adolescentes rurales tienen menores expectativas educativas y ocupacionales que sus pares urbanos.Igualmente, los padres y profesores de los adolescentes rurales, tienen menores expectativas sociales sobre sus hijos o alumnos que sus pares urbanos (Roscigno y Crowley, 2001; Roscigno et al., 2006 ). Las menores expectativas sociales de las zonas rurales suelen ser atribuidas a distintos efectos del aislamiento territorial: mayores tasas de pobreza que 
las zonas urbanas( ; Save the Children, 2002); mayor distancia a los centros educativos medios y superiores (Byun, et al., 2012); ymayordificultad de las escuelas rurales para contratar maestros (; Provasnik et al., 2007).Sin embargo, otros autores consideran que las menores expectativas sociales de los jóvenes rurales no se deben a las desventajas propias del aislamiento territorial, sino a una sociabilidad más densa, propia de las comunidades rurales, que genera fuertes lazos afectivos con la familia, con el resto de la comunidad y con los trabajos agrarios. Como resultado de este proceso, los jóvenes rurales adaptan sus expectativas sociales a la estructura productiva local, evitandoasí emigrar (Elder y Conger, 2000; Elder, King y Conger, 1996; Howley, 2006; ). En este sentido, los jóvenes rurales que tienen altas expectativas sociales, suelen experimentar tensiones afectivas, ya que para realizarlas tendrán que alejarse de sus familias (Corbett, 2009; ; Hektner, 1995). En la misma línea interpretativa, Visscher (2004) afirma en un trabajo sobre Perú,que las altas expectativas educativas, son en muchas ocasiones, una estrategia para abandonar el mundo rural. Los autores también difieren sobre el grado en que se diferencian las expectativas sociales rurales de las urbanas. Haller y Virkler(1993) afirman quela diferencia entre las aspiraciones educativas de los alumnos rurales con los urbanos, una vez controlada la clase social,son muy reducidas y se pueden explicar por las característicasdel mercado laboral local, más diverso en los territorios urbanos,que utilizan los jóvenes como referencia para construir sus expectativas. Por su parte, Hu (2003) considera que las expectativas educativas de alumnos urbanos y rurales sólo divergen a partir de los dieciséis años ( $10^{\circ}$ curso en EE.UU.).En un trabajo más reciente, Howley (2006) afirma que las expectativas educativas y ocupacionales entre alumnos rurales y urbanos de EE.UU., sólo se diferencian significativamente para los estudios de posgrado, atribuyendo dicha diferencia al mayor arraigo territorial de los jóvenes rurales y a que la estructura productiva rural es más homogénea que la urbana. Por último, Williams (2009) informa de diferencias significativas, también en EE.UU., entre las expectativas que albergan los padres rurales y urbanos de que sus hijos alcancen la universidad. Este autor señala además, que la percepción de los jóvenes sobre las expectativas de sus profesores, afecta más en la generación de expectativas propiasa los jóvenes rurales que a sus pares urbanos. Esto nos indicaría que los docentes son más importantes para los alumnos rurales que para los urbanos. Este conjunto de estudios centrados en la ruralidad no recogen, en la mayoría de los casos, las condiciones extremas que impone el aislamiento y, al estar basados en países desarrollados donde las infraestructuras y la conectividad territorial son relativamente densas, podrían minusvalorar las diferencias entre las localidades aisladas de países en desarrollo o subdesarrollados y el resto del territorio.

\section{Localidades aisladas}

La actual Política Nacional de Desarrollo de Localidades Aisladas (PNDLA) del Estado chileno tiene como principios fundamentales la búsqueda de la equidad social y territorial, siendo su objeto principal la identificación, estudio e intervención de las localidades aisladas (Decreto $n^{\circ} 608$, 15 de julio de 2010). Dicha política, entiende por localidades aisladas "todas aquellas porciones de territorio con comunidades humanas, que se encuen- 
tren geográficamente distanciadas de los grandes centros urbanos, y que muestren baja dotación de servicios básicos, que tengan dificultades de conectividad física y baja conectividad digital, que dispongan de muy baja densidad de población, que presenten dispersión en la distribución territorial de sus habitantes y cuenten con baja presencia de servicios públicos” (GORE y SUBDERE, 2012). En 2011, la Subsecretaría de Desarrollo Regional y Administrativo (SUBDERE) del Gobierno de Chile estableció los cuatro criterios que el Estado chileno utiliza para identificar y calificar a una localidad como aislada. Estos son: el físico-geográfico, el de conectividad-movilidad, el de integración sociocultural y el demográfico.Posteriormente, en un estudio realizado por el Gobierno Regional de Antofagasta (GORE) y por la SUBDERE (2012) se utilizaron los siguientes indicadores y variables para identificar a las localidades en situación de aislamiento de la Región de Antofagasta (cuadro 1). Como puede apreciarse en el cuadro 1, los criterios físico-geográfico y de conectividad-movilidad fueron los que tuvieron mayor ponderación (35\% cada uno), mientras que la ponderación más baja (10\%) fue asignada al criterio demográfico.El estudio concluyó que, en la Región de Antofagasta, existen 50 localidades en situación de aislamiento ${ }^{1}$ (GORE y SUBDERE, 2012, p. 55), con una población total aproximada de 7.338 habitantes. Una de estas localidades aisladas es la Caleta Paposo, objeto de nuestro estudio. La localidad de Antofagasta no fue incluida en el análisis, al ser considera la mejor dotada y conectada de la región.

\section{Cuadro 1}

\section{Criterios de aislamiento territorial: ponderaciones, indicadores y variables}

\begin{tabular}{|c|c|c|c|c|}
\hline Criterio & Ponderador & Indicador & $\begin{array}{l}\text { Ponderación } \\
\text { desagregada por } \\
\text { indicador }\end{array}$ & Variable \\
\hline \multirow[t]{4}{*}{$\begin{array}{l}\text { Físico/ } \\
\text { Geográfico }\end{array}$} & \multirow[t]{4}{*}{$35 \%$} & $\begin{array}{l}\text { Distancia a capital } \\
\text { Provincial }\end{array}$ & $25 \%$ & $\begin{array}{l}\text { Tiempo expresado en } \\
\text { minutos a la capital } \\
\text { provincial }\end{array}$ \\
\hline & & $\begin{array}{l}\text { Distancia a capital } \\
\text { Comunal }\end{array}$ & $25 \%$ & $\begin{array}{l}\text { Tiempo expresado en } \\
\text { minutos a la capital } \\
\text { comunal }\end{array}$ \\
\hline & & $\begin{array}{l}\text { Características } \\
\text { Climáticas }\end{array}$ & $25 \%$ & $\begin{array}{l}\text { Su medición utiliza la } \\
\text { determinación de } \\
\text { agresividad climática }\end{array}$ \\
\hline & & Tipología de Acceso & $25 \%$ & $\begin{array}{l}\text { Tipo o vía de acceso a } \\
\text { la localidad }\end{array}$ \\
\hline \multirow[t]{2}{*}{$\begin{array}{l}\text { Conectivida } \\
\text { dy } \\
\text { Movilidad }\end{array}$} & \multirow[t]{2}{*}{$35 \%$} & $\begin{array}{l}\text { Transporte público/ } \\
\text { Frecuencia }\end{array}$ & $50 \%$ & $\begin{array}{l}\text { Frecuencia de } \\
\text { transporte público } \\
\text { hacia las localidades }\end{array}$ \\
\hline & & Conectividad & $50 \%$ & $\begin{array}{l}\text { Cantidad de servicios } \\
\text { de conectividad por } \\
\text { localidad }\end{array}$ \\
\hline \multirow[t]{3}{*}{$\begin{array}{l}\text { Integración } \\
\text { Socio- } \\
\text { cultural }\end{array}$} & \multirow[t]{3}{*}{$20 \%$} & $\begin{array}{l}\text { Equipamiento } \\
\text { Comunitario }\end{array}$ & $30 \%$ & $\begin{array}{l}\text { Cantidad de } \\
\text { equipamiento por } \\
\text { localidad }\end{array}$ \\
\hline & & Servicios & $30 \%$ & $\begin{array}{l}\text { Cantidad de servicios } \\
\text { por localidad }\end{array}$ \\
\hline & & Educación/ Salud & $40 \%$ & $\begin{array}{l}\text { Cantidad de } \\
\text { establecimientos por } \\
\text { localidad }\end{array}$ \\
\hline $\begin{array}{l}\text { Demográfic } \\
\text { o }\end{array}$ & $10 \%$ & Número de Habitantes & $100 \%$ & $\begin{array}{l}\text { Habitantes de cada } \\
\text { localidad }\end{array}$ \\
\hline
\end{tabular}

Fuente: GORE y SUBDERE, 2012, p. 31 
Como podemos observar, ni en la definición de localidades aisladas que nos ofrece la PNDLA, ni en las dimensiones que utiliza el SUBDERE para identificarlas, se contemplan los recursos laborales del territorio, es decir, no se considera la estructura productiva local que configura las oportunidades laborales para sus habitantes. La omisión de una dimensión que capture el grado de diversificación productiva o laboral resulta llamativa porque se suele considerar al empleo como uno de los recursos fundamentales del territorio, ya que su ausencia o precariedad es la principal causa de emigración, comprometiendo gravemente la existencia del mismo. En lo referente a la generación de expectativas sociales, la ausencia de la dimensión productiva o laboral es todavía más grave, ya que ésta las afecta en gran medida.

\section{Contexto socio-territorial de los colegios}

La Región de Antofagasta se encuentra situada en el norte de Chile, en pleno desierto de Atacama. Limita al norte con la Región de Tarapacá, al sur con la Región de Atacama, al noreste con Bolivia, al sureste con Argentina y al oeste con el Océano Pacífico. La Región de Antofagasta está divida en tres provincias administrativas, la provincia de Antofagasta cuya capital es la ciudad de Antofagasta, la provincia de El Loa cuya capital es la ciudad de Calama y la provincia de Tocopilla cuya capital es la ciudad de Tocopilla. En total cuanta con una superficie de $126.049 \mathrm{~km} 2$, que suponen el $17 \%$ de la superficie total de Chile y con una población de 569.634 habitantes, que representa solamente el 3,4\% de la población del país (Casen, 2009). La mayor parte de la población de la región se concentra en la ciudad de Antofagasta con 285.255 habitantes y en la ciudad de Calama 138.402 con habitantes (Censo, 2002). El desierto de Atacama impone un medio inhóspito que ha dificultado históricamente el poblamiento de la región. La aridez del desierto, la altura del altiplano, el tamaño de la región (es la segunda mayor de Chile) y su escaso poblamiento han producido un sistema de asentamientos humanos con gran dispersión territorial, en el que los núcleos urbanos se encuentran muy distanciados entre sí.

En este contexto la ciudad de Antofagasta, por su tamaño, por los servicios públicos y privados que concentra y por su nivel de conectividad, está considerada la localidad mejor integrada territorialmente de la región.Además, Antofagasta destaca por tener una economía muy dinámica derivada de su especialización minera, el 12,7\% de su fuerza laboral trabaja en este rubro, frente al 0,4\% nacional (Casen, 2015). A consecuencia de los anterior, la ciudad presenta un salario promedio de 603.000 pesos chilenos (1.025 US\$) con sólo un 2,7\% de desocupación, frente a un salario de 462.000 pesos (785 US\$) y una desocupación del 4,4\% para el conjunto de Chile (Casen, 2015). La escuela básica D-74 de la ciudad de Antofagasta, situada en la Unidad Vecinal n 18 sector "Hospital Centro", está compuesta por un universo aproximado de 750 alumnos (300 familias aprox.), distribuidos en 19 cursos desde el nivel de transición menor hasta octavo año básico. La planta docente la componen 27 profesores y 3 educadoras de párvulos, se encuentra ubicado en área urbana de dependencia municipal, 
cuyo sostenedor es la Corporación Municipal Desarrollo Social de Antofagasta.

La Caleta Paposo es un caserío que pertenece administrativamente a la comuna de Taltal, aunque se encuentra situado $54 \mathrm{~km}$ al norte de dicha comuna. Paposo alberga a una población aproximada de 350 habitantes (encuesta municipal). Actualmente, la actividad económica predominante es la pesca y la recolección de algas ${ }^{2}$. La escuela básica G-107 cuenta con 55 alumnos aproximadamente (29 familias), distribuidos en 8 cursos desde primero básico a octavo básico. Los recursos docentes de esta escuela están compuestos por 8 profesores. Los estudiantes de este establecimiento educacional provienen en su mayoría de hogares con problemáticas socioeconómicas, manteniéndose sobre la media regional en indigencia y pobreza, evidenciando así familias más vulnerables. El Índice de Vulnerabilidad Escolar de la zona (JUNAEB) muestra problemas de baja escolaridad,desnutrición y pobreza de consumo en los hogares de los estudiantes. En concreto, el número de estudiantes de esta escuela considerados prioritarios ${ }^{3}$ superaba el 66\% en el 2010.

\section{Figura 1 \\ Localidades de estudio}

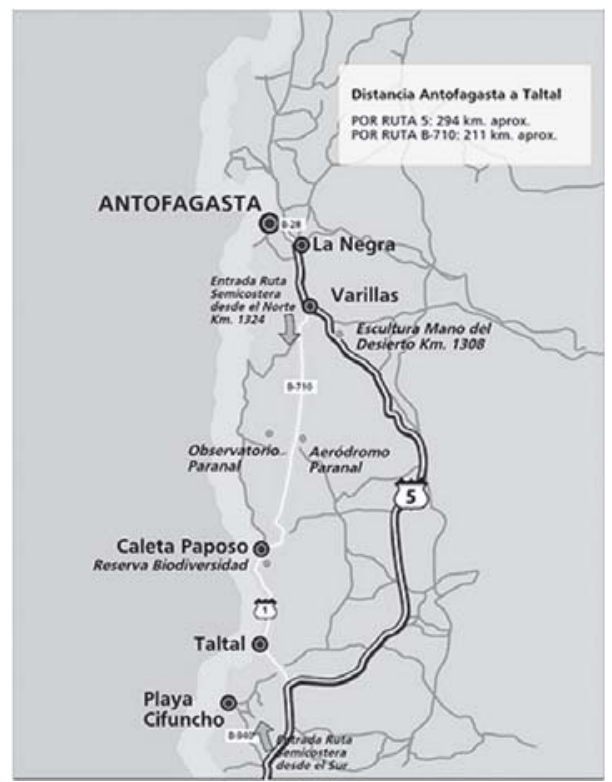

Fuente: Google Maps 


\section{Metodología}

Se realizó una encuesta a la población de madres de alumnos del colegio Paposo $\left(n_{1}=29\right)$ y a una muestra aleatoria simple de madres del colegio de Antofagasta $\left(n_{2}=66\right)$, con el objetivo de analizar los efectos del aislamiento territorial que sufren los habitantes de la caleta Paposo en la generación de las expectativas sociales (educacionales, económicas y espaciales) sobre sus hijos. Utilizamos tres criterios para seleccionar el colegio no aislado: 1) que estuviese ubicado en la localidad mejor conectada e integrada de la región; 2) que las familias de los alumnos perteneciesen a la misma clase social ${ }^{4}$ que las familias de la Caleta Paposo; 3) y que la dirección del colegio permitiese la realización del estudio. La encuesta recogió información sobre las siguientes propiedades familiares:

\section{Cuadro 2}

Estructura del cuestionario

\begin{tabular}{|l|l|l|}
\hline \multicolumn{1}{|c|}{ Propiedades familiares } & \multicolumn{1}{c|}{ Variables } & \multicolumn{1}{c|}{ Medidas } \\
\hline Edad de la madre & Años de la madre & Métrica \\
\hline Estructura familiar & Estado civil de la madre & Nominal \\
\hline Nivel de hacinamiento & $\mathrm{N}^{\circ}$ de personas que viven en la vivienda & Métrica \\
\hline \multirow{4}{*}{ Clase social familiar } & Nivel educativo madre & Ordinal \\
\cline { 2 - 3 } & Nivel educativo pareja de la madre & Ordinal \\
\cline { 2 - 3 } & Ocupación madre & Nominal \\
\cline { 2 - 3 } & Ocupación pareja de la madre & Nominal \\
\cline { 2 - 3 } & Ingreso familiar mensual & Métrica \\
\hline Identificación escolar del hijo & Curso del hijo & Ordinal \\
\hline \multirow{2}{*}{$\begin{array}{l}\text { Expectativas y aspiraciones } \\
\text { sociales sobre su hijo }\end{array}$} & Expectativas educacionales en el largo plazo & Ordinal \\
\cline { 2 - 3 } & Expectativas laborales & Ordinal \\
\cline { 2 - 3 } & Aspiraciones económicas & Métrica \\
\cline { 2 - 3 } & Aspiraciones espaciales & Nominal \\
\hline
\end{tabular}

Elaboración propia.

Para el análisis estadístico descriptivo de los casos se han realizado tablas de frecuencia y tablas de contingencia. Para el análisis estadístico de dependencia se han utilizado dos técnicas multivariantes;el Modelo Lineal General Multivariante (MLGM) y la Regresión Logística (RL).

El MLGM realiza un análisis de regresión lineal múltiple y un análisis de varianza multifactorial para múltiples variables dependientes. El MLGM admite variables métricas y no métricas como independientes y variables métricas como dependientes. Resulta adecuado tanto para modelos equilibrados como para no equilibrados, siendo además robusto a las desviaciones de la normalidad. Utilizaremos este procedimiento para analizar en un solo modelo todas las expectativas sociales, a excepción de las aspiraciones espaciales (variable nominal). La Regresión Logística nos permite realizar un análisis de dependencia usando variables independientes métricas y no métricas para predecir una variable dependiente nominal. Utilizaremos este procedimiento para analizar las aspiraciones espaciales. 
Polis, Revista Latinoamericana, $N^{\circ}$ 50, 2018

Se realizó además un Análisis de Componentes Principales Categórico (ACPC) con las cinco variables asociadas a la clase social familiar, con el objeto de reducirlas a un solo factor (o dimensión) que resuma la información de las variables originales. Dicha reducción, se soporta en la teoría de Bourdieu (1988, 2000, 2001), que afirma que la clase social de una familia (su capital global) está compuesta por el conjunto de capitales (económico, cultural, social y simbólico) que posee ${ }^{5}$.La nueva variable clase social explica el 65,24\% de la varianza de las variables originales (Autovalor $=3,262$ ) y posee un Alfa de Cronbach de 0,867. Esto nos permitirá reducir significativamente el número de variables independientes en el MLGMy en la Regresión Logística.

\section{Resultados}

Comenzaremos describiendo y comparando las condiciones sociales que caracterizan a las familias de ambos colegios. Como podemos observar en el cuadro 3, las madres de Paposo son, en promedio, tres años más jóvenes que las del colegio de Antofagasta. Observamos también que las familias del colegio de Antofagasta tienen más hijos escolarizados (0,3 hijos) que las familias de Paposo. En línea con lo anterior, el nivel de hacinamiento es mayor en las familias del colegio de Antofagasta que en las de Paposo, habiendo 1,65 personas más por vivienda. Finalmente advertimos que las familias del colegio de Antofagasta tienen unos ingresos mensuales significativamente mayores (186.703 pesos chilenos más) que las del colegio Paposo.

\section{Cuadro 3: Edad de la madre, Hacinamiento en el hogar, $\mathrm{N}^{\circ}$ hijos escolarizados e Ingreso Familiar Mensual}

\begin{tabular}{|c|c|c|c|c|c|}
\hline \multicolumn{2}{|r|}{ Colegio } & $\mathrm{N}$ & Mínimo & Máximo & Media \\
\hline \multirow{5}{*}{ Paposo } & Edad de la madre & 28 & 23 & 52 & 35,25 \\
\hline & Hacinamiento & 29 & 2 & 12 & 4,41 \\
\hline & $\begin{array}{l}\mathrm{N}^{\circ} \text { de hijos } \\
\text { escolarizados }\end{array}$ & 29 & 1 & 3 & 1,76 \\
\hline & $\begin{array}{c}\text { Ingreso Familiar } \\
\text { Mensual }\end{array}$ & 29 & 100.000 & 650.000 & 245862,07 \\
\hline & $\mathrm{N}$ válido (según lista) & 28 & & & \\
\hline \multirow{5}{*}{ Antofagasta } & Edad de la madre & 61 & 26 & 72 & 38,13 \\
\hline & Hacinamiento & 65 & 2 & 15 & 6,06 \\
\hline & $\begin{array}{l}\mathrm{N}^{\circ} \text { de hijos } \\
\text { escolarizados }\end{array}$ & 62 & 1 & 4 & 2,05 \\
\hline & $\begin{array}{c}\text { Ingreso Familiar } \\
\text { Mensual }\end{array}$ & 61 & 75.000 & 1.000 .000 & $432.565,57$ \\
\hline & $\mathrm{N}$ válido (según lista) & 53 & & & \\
\hline
\end{tabular}

Fuente: Elaboración propia 
En los cuadros 4 y 5 podemos observar como el nivel educacional de las familias del colegio de Antofagasta es más alto que el de las familias del colegio Paposo. Tanto las madres del colegio Paposo como sus parejas alcanzaron con menor frecuencia los niveles educativos medio y superior.

\section{Cuadro 4 \\ Nivel educacional de la Madre}

\begin{tabular}{|l|l|l|l|l|l|}
\hline \multicolumn{2}{|c|}{} & & & \\
\hline Colegio & & Frecuencia & Porcentaje & $\begin{array}{l}\text { Porcentaje } \\
\text { válido }\end{array}$ & $\begin{array}{l}\text { Porcentaje } \\
\text { acumulado }\end{array}$ \\
\hline \multirow{4}{*}{ Paposo } & Básica & 15 & 51,7 & 51,7 & 51,7 \\
\cline { 2 - 7 } & Media & 10 & 34,5 & 34,5 & 86,2 \\
\cline { 2 - 6 } & Técnica & 3 & 10,3 & 10,3 & 96,6 \\
\cline { 2 - 6 } & Superior & 1 & 3,4 & 3,4 & 100 \\
\cline { 2 - 6 } & Total & 29 & 100 & 100 & \\
\hline \multirow{5}{*}{ Antofagasta } & Básica & 19 & 28,8 & 28,8 & 28,8 \\
\cline { 2 - 6 } & Media & 35 & 53 & 53 & 81,8 \\
\cline { 2 - 6 } & Técnica & 8 & 12,1 & 12,1 & 93,9 \\
\cline { 2 - 6 } & Superior & 4 & 6,1 & 6,1 & 100 \\
\cline { 2 - 6 } & Total & 66 & 100 & 100 & \\
\hline
\end{tabular}

Fuente: Elaboración propia

\section{Cuadro 5:}

Nivel educacional de la Pareja

\begin{tabular}{|c|c|c|c|c|c|c|}
\hline \multicolumn{3}{|l|}{ Colegio } & Frecuencia & Porcentaje & $\begin{array}{l}\text { Porcentaje } \\
\text { válido }\end{array}$ & $\begin{array}{l}\text { Porcentaje } \\
\text { acumulado }\end{array}$ \\
\hline \multirow{6}{*}{ Paposo } & & Básica & 19 & 65,5 & 65,5 & 65,5 \\
\hline & & Media & 4 & 13,8 & 13,8 & 79,3 \\
\hline & & Técnica & 2 & 6,9 & 6,9 & 86,2 \\
\hline & & Superior & 1 & 3,4 & 3,4 & 89,7 \\
\hline & & $\mathrm{NS} / \mathrm{NC}$ & 3 & 10,3 & 10,3 & 100,0 \\
\hline & & Total & 29 & 100,0 & 100,0 & \\
\hline \multirow{8}{*}{ Antofagasta } & & Básica & 10 & 15,2 & 15,9 & 15,9 \\
\hline & & Media & 35 & 53,0 & 55,6 & 71,4 \\
\hline & & Técnica & 6 & 9,1 & 9,5 & 81,0 \\
\hline & & Superior & 9 & 13,6 & 14,3 & 95,2 \\
\hline & & $\mathrm{NS} / \mathrm{NC}$ & 3 & 4,5 & 4,8 & 100,0 \\
\hline & & Total & 63 & 95,5 & 100,0 & \\
\hline & Perdidos & Sistema & 3 & 4,5 & & \\
\hline & \multicolumn{2}{|l|}{ Total } & 66 & 100,0 & & \\
\hline
\end{tabular}

Fuente: Elaboración propia 
Polis, Revista Latinoamericana, $N^{\circ}$ 50, 2018

Respecto a la ocupación, podemos ver en cuadro 6 como las madres del colegio de Antofagasta están más integradas en el mercado laboral que las madres de Paposo (15\% más de trabajadoras activas), teniendo además una mayor participación en los trabajos cualificados (9,8\%). De forma inversa, advertimos cómo las parejas de las mujeres de Paposo (cuadro 7), tienen unas ocupaciones ligeramente más cualificadas que sus pares del colegio de Antofagasta (un 7\% más de trabajadores cualificados).

\section{Cuadro 6:}

Trabajo de la Madre

\begin{tabular}{|c|c|c|c|c|c|c|}
\hline \multicolumn{3}{|l|}{ Colegio } & \multirow{2}{*}{\begin{tabular}{|l|} 
Frecuencia \\
19 \\
\end{tabular}} & \multirow{2}{*}{\begin{tabular}{|l|} 
Porcentaje \\
65,5 \\
\end{tabular}} & \multirow{2}{*}{\begin{tabular}{|l|}
$\begin{array}{l}\text { Porcentaje } \\
\text { válido }\end{array}$ \\
65,5
\end{tabular}} & \multirow{2}{*}{$\begin{array}{l}\begin{array}{l}\text { Porcentaje } \\
\text { acumulado }\end{array} \\
65,5\end{array}$} \\
\hline \multirow{3}{*}{ Paposo } & & Dueña de casa & & & & \\
\hline & & $\begin{array}{l}\text { Trabajo No } \\
\text { Cualificado }\end{array}$ & 10 & 34,5 & 34,5 & 100,0 \\
\hline & & Total & 29 & 100,0 & 100,0 & \\
\hline \multirow{6}{*}{ Antofagasta } & & Dueña de casa & 31 & 47,0 & 50,8 & 50,8 \\
\hline & & $\begin{array}{l}\text { Trabajo No } \\
\text { Cualificado }\end{array}$ & 24 & 36,4 & 39,3 & 90,2 \\
\hline & & Trabajo Cualificado & 6 & 9,1 & 9,8 & 100,0 \\
\hline & & Total & 61 & 92,4 & 100,0 & \\
\hline & Perdidos & Sistema & 5 & 7,6 & & \\
\hline & \multicolumn{2}{|l|}{\begin{tabular}{|l|} 
Total \\
\end{tabular}} & 66 & 100,0 & & \\
\hline
\end{tabular}

Fuente: Elaboración propia

Cuadro 7:

Trabajo de la Pareja

\begin{tabular}{|l|l|l|l|l|l|l|}
\hline \multicolumn{2}{|l|}{ Colegio } & Frecuencia & Porcentaje & $\begin{array}{l}\text { Porcentaje } \\
\text { válido }\end{array}$ & $\begin{array}{l}\text { Porcentaje } \\
\text { acumulado }\end{array}$ \\
\hline \multirow{4}{*}{ Paposo } & \multirow{4}{*}{$\begin{array}{l}\text { Trabajo No } \\
\text { Cualificado }\end{array}$} & 15 & 51,7 & 51,7 & 51,7 \\
\cline { 3 - 7 } & Trabajo Cualificado & 8 & 27,6 & 27,6 & 79,3 \\
\hline & NS/NC & 6 & 20,7 & 20,7 & 100,0 \\
\cline { 3 - 7 } & Total & 29 & 100,0 & 100,0 & \\
\hline \multirow{4}{*}{ Antofagasta } & $\begin{array}{l}\text { Trabajo No } \\
\text { Cualificado }\end{array}$ & 28 & 42,4 & 58,3 & 58,3 \\
\cline { 3 - 7 } & Trabajo Cualificado & 10 & 15,2 & 20,8 & 79,2 \\
\cline { 3 - 7 } & NS/NC & 10 & 15,2 & 20,8 & 100,0 \\
\cline { 3 - 7 } & Total & 48 & 72,7 & 100,0 & \\
\cline { 2 - 7 } & Perdidos & Sistema & 18 & 27,3 & & \\
\cline { 2 - 7 } & Total & 66 & 100,0 & & \\
\hline
\end{tabular}

Fuente: Elaboración propia

Por último nos referiremos en la estructura de las familias de los alumnos. Como podemos ver en el cuadro 8 , el 44,8\% de las familias de Paposo son monoparentales maternas (separadas/divorciadas + viudas + solteras), mientras que en el colegio de Antofagasta son el 31,8\% de 
las familias la que se encuentran en esta situación de mayor vulnerabilidad social.

\section{Cuadro 8: \\ Estado Civil ${ }^{6}$}

\begin{tabular}{|l||l|l|l|l|l|}
\hline \multicolumn{2}{|c|}{} & Frecuencia & Porcentaje & $\begin{array}{l}\text { Porcentaje } \\
\text { válido }\end{array}$ & $\begin{array}{l}\text { Porcentaje } \\
\text { acumulado }\end{array}$ \\
\hline \multirow{5}{*}{ Colegio } & Casada & 7 & 24,1 & 24,1 & 24,1 \\
\cline { 2 - 7 } & Separada o divorciada & 1 & 3,4 & 3,4 & 27,6 \\
\cline { 2 - 7 } & Convive con pareja & 9 & 31,0 & 31,0 & 58,6 \\
\cline { 2 - 7 } & Viuda & 1 & 3,4 & 3,4 & 62,1 \\
\cline { 2 - 7 } & Soltera & 10 & 34,5 & 34,5 & 96,6 \\
\cline { 2 - 7 } & NS/NC & 1 & 3,4 & 3,4 & 100,0 \\
\cline { 2 - 7 } & Total & 29 & 100,0 & 100,0 & \\
\hline \multirow{5}{*}{ Antofagasta } & Casada & 32 & 48,5 & 48,5 & 48,5 \\
\cline { 2 - 7 } & Separada o divorciada & 6 & 9,1 & 9,1 & 57,6 \\
\cline { 2 - 7 } & Convive con pareja & 13 & 19,7 & 19,7 & 77,3 \\
\cline { 2 - 7 } & Viuda & 2 & 3,0 & 3,0 & 80,3 \\
\cline { 2 - 7 } & Soltera & 13 & 19,7 & 19,7 & 100,0 \\
\cline { 2 - 6 } & Total & 66 & 100,0 & 100,0 & \\
\hline
\end{tabular}

Fuente: Elaboración propia

En resumen, podemos decir que las familias del colegio de Antofagasta tienenmejorescondiciones sociales que las familias de Paposo, ya que poseen mayor nivel educacional, mayor tasa de actividad femenina, mayor probabilidad de ser familias biparentales y mayores ingresos que las familias de Paposo. La única ventaja clara que presentan éstas últimas, es un menor nivel de hacinamiento. Por tanto, aunque ambos grupos sociales pueden ser catalogados como vulnerables (ambos están en el primer quintil de ingresos de la región), la situación de la comunidad educativa de Paposo es significativamente más precaria.

\section{Expectativas sociales de las madres}

A continuación expondremos los resultados obtenidos en el análisis de las expectativas sociales que tienen las madres sobre sus hijos. En siguientes tablas podemos examinar las expectativas ${ }^{7}$ educativas en el largo plazo (cuadro 9). Como podemos advertir, las madres de Paposo tienen una mayor creencia de que sus hijos alcanzarán la universidad, aunque las diferencias son pequeñas (sólo 3,6\%). 


\section{Cuadro 9: \\ Expectativas educativas a largo plazo}

\begin{tabular}{|c|c|c|c|c|c|c|c|}
\hline & & \multicolumn{5}{|c|}{ Creo que podrá estudiar hasta... } & \multirow[t]{2}{*}{ Total } \\
\hline & & EGB & $\begin{array}{|ll|}\text { Liceo } & \circ \\
\text { Politécnico } & \end{array}$ & $\begin{array}{l}\text { Centro de } \\
\text { Formación } \\
\text { Técnica }\end{array}$ & Universidad & NS/NR & \\
\hline \multirow{2}{*}{ Colegio } & Paposo & $6,9 \%$ & $6,9 \%$ & $10,3 \%$ & $72,4 \%$ & $3,4 \%$ & $100,0 \%$ \\
\hline & Antofagasta & $3,1 \%$ & $7,8 \%$ & $18,8 \%$ & $68,8 \%$ & $1,6 \%$ & $100,0 \%$ \\
\hline \multicolumn{2}{|l|}{ Total } & $4,3 \%$ & $7,5 \%$ & $16,1 \%$ & $69,9 \%$ & $2,2 \%$ & $100,0 \%$ \\
\hline
\end{tabular}

Fuente: Elaboración propia

Respecto a las expectativas laborales, podemos observar en el cuadro 10 cómo las madres de Paposo afirman tener mayor seguridad que las madres del colegio de Antofagasta, de que sus hijos encuentren un trabajo relacionado con sus estudios (69\% vs. 58,5\%). Aunque la diferencia entre los dos grupos de madres no es alta $(10,5 \%)$ podría ser significativa.

\section{Cuadro 10: \\ Expectativas laborales\% dentro de Colegio}

\begin{tabular}{|c|c|c|c|c|c|c|c|}
\hline & & \multicolumn{5}{|c|}{ ¿Cree que encontrará un trabajo relacionado con lo que estudió? } & \multirow[t]{2}{*}{ Total } \\
\hline & & Sí, seguro & $\begin{array}{l}\mathrm{Si} \text {, pero no } \\
\text { relacionado con } \\
\text { mis estudios }\end{array}$ & $\begin{array}{l}\text { Puede que no } \\
\text { encuentre } \\
\text { inmediatamente }\end{array}$ & $\begin{array}{l}\text { No, estará sin } \\
\text { trabajo }\end{array}$ & NS/NR & \\
\hline \multirow{2}{*}{ Colegio } & Paposo & $69,0 \%$ & $17,2 \%$ & $3,4 \%$ & $10,3 \%$ & & $100,0 \%$ \\
\hline & Antofagasta & $58,5 \%$ & $23,1 \%$ & $10,8 \%$ & $1,5 \%$ & $6,2 \%$ & $100,0 \%$ \\
\hline \multicolumn{2}{|l|}{ Total } & $61,7 \%$ & $21,3 \%$ & $8,5 \%$ & $4,3 \%$ & $4,3 \%$ & $100,0 \%$ \\
\hline
\end{tabular}

Fuente: Elaboración propia

En cuanto a deseo de emplazamiento futuro de los hijos (cuadro 11), observamos como las madres de Paposo anhelan en mayor medida que las del colegio de Antofagasta, que sus hijos se establezcan en otro municipio (83,3\% vs. 11,9\%), siendo esta diferencia muy notable. Entendemos por ello que las madres de Paposo perciben que su municipio carecerá de lo necesario para generar el bienestar social que desean para sus hijos. Por su lado, el deseo de migración de los hijos, es muy bajo en las madres del colegio de Antofagasta, pues deben de creer que la ciudad de Antofagasta será capaz de satisfacer las necesidades de sus hijos. 


\section{Cuadro 11: \\ Aspiraciones espaciales \% dentro de Colegio}

\begin{tabular}{|c|c|c|c|c|c|}
\hline & & \multicolumn{3}{|c|}{ ¿Dónde le gustaría que viva cuando sea adulto? } & \multirow[t]{2}{*}{ Total } \\
\hline & & $\begin{array}{l}\text { Mismo } \\
\text { municipio }\end{array}$ & Otro Municipio & NS/NR & \\
\hline \multirow{2}{*}{ Colegio } & Paposo & $16,7 \%$ & $83,3 \%$ & & $100,0 \%$ \\
\hline & Antofagasta & $55,9 \%$ & $11,9 \%$ & $32,2 \%$ & $100,0 \%$ \\
\hline \multicolumn{2}{|l|}{ Total } & $44,6 \%$ & $32,5 \%$ & $22,9 \%$ & $100,0 \%$ \\
\hline
\end{tabular}

Fuente: Elaboración propia

Finalmente (cuadro 12) comprobamos que las aspiraciones económicas que las madres de Paposo realizan sobre sus hijos, son sensiblemente más bajas que las que tienen las madres del colegio de Antofagasta (507.407 vs. 747.059 pesos chilenos). Esta diferencia tan abultada (-239.652 pesos) podría estar explicada tanto por la composición social de los colegios ${ }^{8} \mathrm{como}$ por las características propias del territorio ${ }^{9}$. Aclararemos este punto más adelante.

\section{Cuadro 12: Aspiraciones económicas}

\begin{tabular}{|l|l|l|l|l|l|l|}
\hline Colegio & N & Mínimo & Máximo & Media & Desv. típ. \\
\hline \multirow{4}{*}{ Paposo } & $\begin{array}{l}\text { ¿Cuánta plata le gustaría que } \\
\text { ganase, como mínimo, su hijo } \\
\text { al mes? }\end{array}$ & 27 & 200000 & 900000 & 507.407 & 177971,316 \\
\cline { 2 - 7 } & Nválido (según lista) & 27 & & & & \\
\hline \multirow{3}{*}{ Antofagasta } & $\begin{array}{l}\text { ¿Cuánta plata le gustaría que } \\
\text { ganase, como mínimo, su hijo } \\
\text { al mes? }\end{array}$ & 51 & 200000 & 2000000 & 747.059 & 410537,668 \\
\cline { 2 - 7 } & N válido (según lista) & 51 & & & & \\
\hline
\end{tabular}

Fuente: Elaboración propia

A continuación, realizaremos un Modelo Lineal General Multivariante para conocer qué características de las familias inciden significativamente en las expectativas sociales que las madres generan sobre sus hijos, prestando especial atención al aislamiento territorial (pertenecer a la caleta Paposo). Como podemos observar (cuadro 13), en las expectativas educacionales a largo plazo ${ }^{10}$ (primera sección) sólo aparecen significativamente afectadas por la edad de la madre. Cuanto mayor es la madre, peores expectativas tienen sobre sus hijos.

Respeto a las expectativas laborales que las madres tienes sobre sus hijos ${ }^{11}$ (segundasección), sólo la variable hijos escolarizados presenta un cierto impacto, aunque poco significativo. A mayor número de hijos escolarizados, mejores expectativas laborales sobre el hijo. 
Por último (tercerasección) encontramos que las variables colegio y clase social inciden significativamente, y con similar intensidad $\left(t_{\text {colegio }}=-\right.$ 2,741 vs. $t_{\text {clase }}=2,852$ ), en las aspiraciones económicas que las madres tienen sobre sus hijos. Comprobamos que las madres de Paposo desean para sus hijos un salario inferior (-351.283 pesos) al de las madres del colegio de Antofagasta. Por otro lado, el incremento de la clase social familiar, genera un aumento en las aspiraciones económicas sobre los hijos.

\section{Cuadro 13:}

Estimaciones de los parámetros (MLGM)

\begin{tabular}{|c|c|c|c|c|c|}
\hline Variable depen diente & & $\mathbf{B}$ & Errortip. & $t$ & Sig. \\
\hline $\begin{array}{l}\text { Creo que podrá estudiar hasta.. } \\
\text { expectativa educacionsl largo plaso } \\
R \text { cuadrado corregida }=, 188\end{array}$ & $\begin{array}{l}\text { Paposo } \\
\text { Antofagasta } \\
\text { Biperental } \\
\text { Monoparental } \\
\text { matems } \\
\text { Edad madre } \\
\text { Clase social } \\
\text { Hacinamiento } \\
\text { Hijos } \\
\text { escolarizados } \\
\text { Curso }\end{array}$ & $\begin{array}{l}-, 185 \\
0^{2} \\
, 077 \\
0^{2} \\
-, 050 \\
, 082 \\
-, 007 \\
, 161 \\
-, 038\end{array}$ & $\begin{array}{l}, 275 \\
, 206 \\
, 018 \\
, 065 \\
, 055 \\
, 137 \\
, 056\end{array}$ & $\begin{array}{l}-2,721 \\
1,250 \\
-, 134 \\
1,175 \\
-, 681\end{array}$ & $\begin{array}{l}, 504 \\
, 712 \\
, 01044 \\
, 219 \\
, 894 \\
, 247 \\
, 500\end{array}$ \\
\hline $\begin{array}{l}\text { ¿Cres que encontrará un trabajo } \\
\text { relacionado con lo que estudió? } \\
\text { expectativa laboral } \\
R \text { cuadrado corregida }=, 010\end{array}$ & $\begin{array}{l}\text { Intersacción } \\
\text { Paposo } \\
\text { Antofagasta } \\
\text { Biperental } \\
\text { Monoparental } \\
\text { matema } \\
\text { Edad madre } \\
\text { Clase social } \\
\text { Hacinamiento } \\
\text { Hijos } \\
\text { escolarizados } \\
\text { Curso }\end{array}$ & \begin{tabular}{|l|}
1,104 \\
,- 425 \\
$0^{2}$ \\
, 239 \\
$0^{2}$ \\
, 029 \\
,- 087 \\
, 008 \\
,- 282 \\
,- 043
\end{tabular} & $\begin{array}{l}, 848 \\
, 334 \\
, 250 \\
, 022 \\
, 079 \\
, 067 \\
, 166 \\
, 068\end{array}$ & \begin{tabular}{|l|}
1,302 \\
$-1,274$ \\
, 956 \\
1,290 \\
$-1,091$ \\
, 117 \\
$-1,696$ \\
,- 638 \\
\end{tabular} & $\begin{array}{l}, 201 \\
, 210 \\
, 345 \\
\\
, 204 \\
, 282 \\
, 908 \\
, 0984 \\
, 527\end{array}$ \\
\hline $\begin{array}{l}\text { ¿Cuánts plats le guataría que ganse, } \\
\text { como mínimo, su hijo al mes? } \\
\text { sapiración económica } \\
\text { R cuadrado corregida }=, 323\end{array}$ & $\begin{array}{l}\text { Intersacción } \\
\text { Paposo } \\
\text { Antofagasta } \\
\text { Biperentsl } \\
\text { Monoparental } \\
\text { matems } \\
\text { Edad madre } \\
\text { Clase social } \\
\text { Hacinamiento } \\
\text { Hijos } \\
\text { escolarizados } \\
\text { Curso }\end{array}$ & $\begin{array}{l}805554,316 \\
-351282,871 \\
0^{2} \\
122111,092 \\
0^{2} \\
7429,894 \\
86942,903 \\
-32172,030 \\
-13707,939 \\
-22935,020 \\
\end{array}$ & $\begin{array}{l}325431,046 \\
128146,689 \\
95999,836 \\
\\
8584,759 \\
30488,542 \\
25770,670 \\
63768,889 \\
25967,355\end{array}$ & $\begin{array}{l}2,475 \\
-2,741 \\
1,272 \\
\\
, 865 \\
2,852 \\
-1,248 \\
-, 215 \\
-, 883\end{array}$ & $\begin{array}{l}, 018^{44} \\
, 009^{44} \\
, 211 \\
, 392 \\
, 007^{44} \\
, 219 \\
, 831 \\
, 383\end{array}$ \\
\hline
\end{tabular}

Fuente: Elaboración propia 
Para conocer las variables que inciden en las aspiraciones espaciales de las madres sobre sus hijos utilizaremos una Regresión Logística (RL), ya que esta variable dependiente es nominal. Podemos apreciar como el colegio es la única variable independiente que resulta ser claramente significativa para las aspiraciones espaciales (cuadro 14). Es mucho más probable (30,75 veces) que las madres de Paposo prefieran que sus hijos emigren, a que lo deseen las madres del colegio de Antofagasta.

\section{Cuadro 14: Estimaciones de los parámetros (RL)}

\begin{tabular}{|c|c|c|c|c|c|c|c|}
\hline \multicolumn{2}{|c|}{$\begin{array}{l}\text { ¿Dónde le gustaría que viva su hijo } \\
\text { cuando sea adulto? } \\
\text { Aspiración espacial }\end{array}$} & B & $\begin{array}{l}\text { Error } \\
\text { típ. }\end{array}$ & Wald & gl & Sig. & $\operatorname{Exp}(B)$ \\
\hline \multirow{10}{*}{ Otro municipio } & Intersección & $-1,055$ & 2,438 &, 187 & 1 & ,665 & \\
\hline & Edad madre &, 038 & 054 & 500 & 1 & , 480 & 1,039 \\
\hline & \begin{tabular}{|l|} 
Hacinamiento \\
\end{tabular} &,- 088 & ,279 & ,098 & 1 & ,754 & 916 \\
\hline & Hijos escolarizados &,- 105 &, 613 &, 029 & 1 & 864 & ,901 \\
\hline & \begin{tabular}{|l} 
Curso \\
\end{tabular} &,- 174 & 207 &, 713 & 1 &, 399 &, 840 \\
\hline & Clase social & 1,372 & ,877 & 2,446 & 1 &, 118 & 3,944 \\
\hline & Paposo & 3,426 & 1,082 & 10,035 & 1 &, 002 & 30,756 \\
\hline & Antofagasta & $0^{b}$ & & & 0 & & \\
\hline & Biparental &,- 575 &, 880 & 427 & 1 & ,513 & ,563 \\
\hline & Monoparental & $0^{\mathrm{b}}$ & & & 0 & & \\
\hline
\end{tabular}

Fuente: Elaboración propia

En resumen, los resultados obtenidos por estos dos modelos (MLGM y RL) señalan que la variable colegio, que en este trabajo indica la diferencia territorial (aislamiento vs. no aislamiento), tiene una incidencia significativa en las expectativas sociales que las madres tienen sobre sus hijos, incluso después de controlar por el resto de propiedades familiares. Concretamente, las madres de Paposo (territorio aislado) tienen mejores expectativas educacionales en el largoplazo, menores aspiraciones de ingreso y un mayor deseo de que sus hijosemigren que las madres del colegio de Antofagasta(territorio no aislado).

Hemos visto además como las madres con más hijos escolarizados tienen mejores expectativas sociales (educacionales y laborales) sobre sus hijos. Este hechopodría explicarse por un proceso de disonancia cognitiva (Festinger, 1975), mediante el cual, las madres que hacen un mayor esfuerzo para que sus hijos estudien, porque tienen más hijos escolarizados o porque no tienen pareja, necesitan tener mayores expectativas sociales sobre ellos. La edad de la madre también afecta claramente las expectativas sociales sobre sus hijos. Cuanto mayor es la madre, peores son sus expectativas educaciones en el largo plazo sobre sus hijos. Este hecho podría estar explicado porexperiencias negativas previas que las madres de más edad han tenido con sus hijos mayores, o dicho de otra forma, por la inexperiencia de las madres más jóvenes. 
Finalmente, la variable clase social se comporta en el sentido esperado, esto es, aumentando las expectativas sociales sobre los hijos, aunque en nuestro caso, sólo es claramente significativa para las aspiraciones económicas.

\section{Conclusiones}

En este trabajo hemos podido comprobar que el aislamiento territorial afecta significativamente a la generación de las expectativas sociales que las madres tienes sobre sus hijos adolescentes, incluso controlando esta relación por otros factores relevantes como la clase social, la estructura familiar o la edad de la madre. Sin embargo, el resultado más importante puede que sea el haber desvelado las expectativas de residencia que albergan las madres sobre sus hijos. Como hemos visto, el 83,3\% de las madres de la Caleta Paposo (territorio aislado) esperan que sus hijos emigren y fijen su residencia en otra localidad. Esta expectativa de emigración es 30,7 veces menos probable para las madres de Antofagasta (territorio no aislado).

Estos resultados podrían estar avisándonos de un potencial problema de política pública. Si la intervención del Estado en los territorios aislados se reduce a la mejora de su conectividad y de sus servicios públicos, olvidando la transformación de su estructura productiva, el resultado podría ser la emigración de las generaciones más jóvenes y la posterior desaparición de estas localidades, obteniendo así un efecto opuesto al declarado. Las mejoras en la conectividad reducen los costes de la migración y la mejora de los servicios públicos, especialmente en educación, podrían aumentan las expectativas ocupacionales de los estudiantes. Si estos cambios no se acompañan del desarrollo de la estructura productiva local, para que mediante su diversificación pueda ofrecer oportunidades laborales más atractivas a los jóvenes, éstos podrían acabar abandonando el territorio. Por lo anterior, nos parece necesario que la definición y medición del aislamiento territorial contemple su estructura productiva y el aumento de las oportunidades de empleo, y en consecuencia, que las políticas públicas dirigidas a superarlo o reducirlo, se planteen objetivos de desarrollo y diversificación productiva. De lo contrario, las políticas públicas para los territorios aislados, podrían tener como resultado la creación o el incremento de la brecha entre las expectativas sociales de sus habitantes y las oportunidades laborales objetivas que ofrece el territorio. 


\section{Notas}

${ }^{1}$ Publicado en la ORDE. 001391 el 17 de Julio del 2012.

${ }^{2}$ Lamentablemente no existe ninguna encuesta laboral que considere esta caleta, por lo que no se pueden ofrecer cifras de su estructura ocupacional o de ingresos. Sin embargo, la observación directa de la caleta permite afirmar que las ocupaciones mayoritarias son las ya citas.

${ }^{3}$ Ley $\mathrm{n}^{\circ}$ 20. 248 Subvención Escolar Preferencial para Niños y Niñas Prioritarios (2008). Fue modificada en el 2011 (Ley ${ }^{\circ}$ 20.550).

${ }^{4}$ Se trataba de reducir en lo posible la brecha socioeconómica entre las familias de ambos colegios, aunque las técnicas estadísticas utilizadas permitieroncontrolar el efecto de la clase social y diferenciarlo del efecto producido por el aislamiento territorial.

${ }^{5}$ Una reciente línea de investigación propuesta por Gregory Clark (2014) también entiende estas variables (nivel educativo de los padres y abuelos, estatus ocupacional, ingresos, etc.) como indicadores de la posición socioeconómica subyacente (factor lactante) de la familia de origen. Por lo que la identificación de dicho factor latente es mejor cuanto más indicares se utilicen, debido al error de medición de cada indicador.

${ }^{6}$ La variable "estado civil” se recodificará como dicotómica para los análisis de dependencia. La nueva variable "estructura familiar” se compondrá de dos categorías: “familias monoparentales maternas” (solteras, separadas y viudas) y "familias biparentales (casada y convive en pareja).

${ }^{7}$ Por "expectativa" nos referimos a la probabilidad subjetiva de que ocurra un suceso, esto es, lo que el sujeto cree que ocurrirá. Mientras que utilizaremos el concepto "aspiración" para referirnos a la deseo de que ocurra un determinado suceso sin evaluar sus posibilidades, es decir, lo que el sujeto desea que ocurra.

${ }^{8}$ Hipótesis de clase social: Las familias del colegio de Antofagasta tienen en promedio una posición social superior a las familias de Paposo, por lo que tendrían unas expectativas económicas mayores sobre sus hijos generadas por su clase social.

${ }^{9}$ Hipótesis territorial: El aislamiento territorial que sufren las familias de Paposo, mediante algunos de los mecanismos señalados por la literatura (menor acceso a la educación; estructura productiva menos compleja; o relaciones sociales más densas), generaría expectativas económicas inferiores a las producidas por los territorios no aislados (Antofagasta).

${ }^{10}$ En esta variable: 1 = educación básica; 2 = educación media; 3 = educación técnica y 4 = educación universitaria.

${ }^{11}$ En esta variable: 1 = total seguridad de que trabajará y 4 = total seguridad de que no trabajará. 


\section{Bibliografía}

Bandura, A., Barbaranelli, C., Caprara, G. V., \& Pastorelli, C. (2001).Selfefficacy beliefs as shapers of children's aspirations and career trajectories.Child Development,72(1), 187-206.

Beal, S. J., \& Crockett, L. J. (2010).Adolescents occupational and educational aspirations and expectations: Links to high school activities and adult educational attainment,Developmental Psychology, 46(1), 258-265.

Beck, U. (1998). La sociedad del riesgo: hacia una nueva modernidad.Barcelona, España:Paidós ibérica.

Boudon, R. (1973). Educación y movilidad social: un modelo estructural.Educación y Sociedad, 2, 173-185.

Bourdieu, P. (1988).La distinción. Criterios y bases sociales del gusto. Madrid, España:Taurus.

Bourdieu, P. (2000). Cuestiones de sociología (No. 166).Madrid, España: Ediciones AKAL.

Bourdieu, P. (2001). Poder, derecho y clases sociales. Bilbao, España: Desclée.

Bourdieu, P. y Passeron, C. (1981).La reproducción. Elementos para una teoría del sistema de enseñanza.Barcelona, España: Laia.

Byun, S. Y., Meece, J. L., \& Irvin, M. J. (2012),Rural-nonrural disparities in postsecondary educational attainment revisited, American educational research journal, 49(3), 412-437.

Byun, S. Y., Meece, J. L., Irvin, M. J., \& Hutchins, B. C. (2012). The Role of Social Capital in Educational Aspirations of Rural Youth. Rural sociology,77(3), 355-379.

Clark, G. (2014). The son also rises: Surnames and the history of social mobility.New Jersey, EUA:Princeton University Press.

Corbett, M. (2009). Rural schooling in mobile modernity: returning to the places I've been. Journal of Research in Rural Education, 24(7), 1-13.

Criado, E. M.(2004). De la reproducción al campo escolar. En Alonso, L. E., Criado, E. M., \& Pestaña, J. L. M. (Eds.).Pierre Bourdieu: las herramientas del sociólogo, 279.Madrid, España: Editorial Fundamentos.

Decreto ${ }^{\circ}$ 608, 15 de julio de 2010. Recuperado de http://www.leychile.cl/ Navegar?idNorma $=1019885$ 
Eccles, J. S., Vida, M. N., \& Barber, B. (2004).The relation of early adolescents' college plans and both academic ability and task-value beliefs to subsequent college enrollment,Journal of Early Adolescence, 24(1), 63-77.

Elder Jr, G. H. (1996). Attachment to place and migration prospects: a developmental perspective, Journal of Research on Adolescence, 6(4), 397-425.

Elder, G. H., \& Conger, R. D. (2000). Children of the land: Adversity and success in rural America.Chicago, EUA: University of Chicago Press.

Festinger, L. (1957). A theory of cognitive dissonance.Stanford, EUA: Stanford University Press,

GORE y SUBDERE, (2012): Política regional para la integración de localidades aisladas: Implementación de ejes estratégicos para la atención de los factores condicionantes del aislamiento en la Región de Antofagasta, Región de Antofagasta 2012-2020. Recuperado de http:/ /www.subdere.gov.cl/sites/default/files/documentos/ politica_localidades_aisladas_antofagasta.pdf

Haller, E., \& Virkler, S. (1993). Another look at rural-nonrural differences in students' educational aspirations,Journal of Research in Rural Education,9(3), 170-178.

Hektner, J. (1995). When Moving Up Implies Moving Out: Rural Adolescent Conflict in the Transition to Adulthood,Journal of Research in Rural Education 11(1):3-14.

Howley, C. W. (2006). Remote possibilities: Rural children's educational aspirations, Peabody Journal of Education, 81(2), 62-80.

Hu, S. (2003). Educational Aspirations and Postsecondary Access and Choice: Students in Urban, Suburban, and Rural Schools Compared, Education policy analysis archives, 11(14), n14.

JUNAEB. Recuperado dehttp://www.junaeb.cl/ive

Kirkpatrick Johnson, M., Elder, G. H., \& Stern, M. (2005). Attachments to family and community and the young adult transition of rural youth. Journal of Research on Adolescence, 15(1), 99-125.

Lichter, D. T., \& Johnson, K. M. (2007). The Changing Spatial Concentration of America's Rural Poor Population, Rural Sociology, 72(3), 331-358.

Monk, D. H. (2007). Recruiting and retaining high-quality teachers in rural areas. The Future of Children, 17(1), 155-174. 
Nadel, W., \& Sagawa, S. (2002). America’s Forgotten Children: Child Poverty in Rural America. Recuperado de: http://www. savethechildren. org/afc/afc_pdf_02. Shtml

Padilla Carmona, M. T., Suárez Ortega, M., \& García Gómez, S. (2009). Elecciones educativas y vocacionales de las chicas onubenses que finalizan la ESO: comparación de sus expectativas con las de sus docentes y familias.Revista de Educación, (9), 75-94.

Provasnik, S., KewalRamani, A., Coleman, M. M., Gilbertson, L., Herring, W., \& Xie, Q. (2007). Status of Education in Rural America. NCES 2007-040. National Center for Education Statistics.

Roscigno, V. J., \& Crowle, M. L. (2001). Rurality, Institutional Disadvantage, and Achievement/Attainment, Rural Sociology, 66(2), 268-292.

Roscigno, V. J., Tomaskovic-Devey, D., \& Crowley, M. (2006). Education and the inequalities of place, Social Forces, 84(4), 2121-2145.

Sen, A. K. (1985).On economic inequality. Nueva Delhi, India: Oxford University Press.

Sen, A. K. (1995).Nuevo examen de la desigualdad, Madrid, España: Alianza Editorial.

SUBDERE (2011).Estudio identificación de territorios aislados 2011. División de Políticas y Estudios, Departamento de Estudios y Evaluación, Unidad de Análisis Territorial, Gobierno de Chile. Recuperado de http://www.subdere.gov.cl/sites/default/files/documentos/web.pdf

Williams, K. K. (2009). From aspirations to access: the role of place in the facilitators of and barriers to postsecondary education attendance (Doctoral dissertation, Ball State University). 